
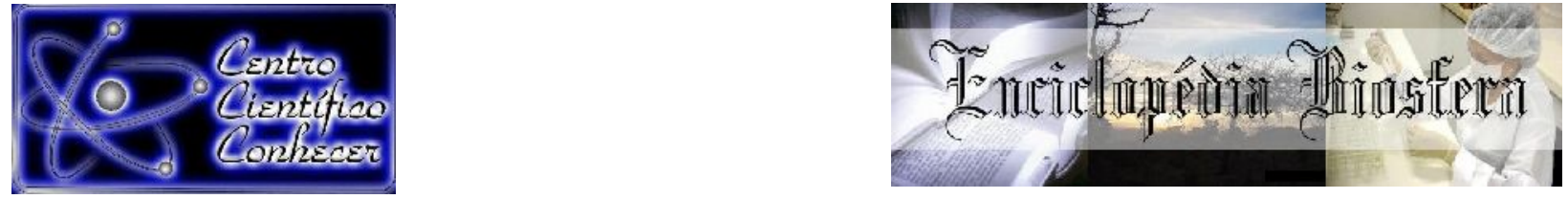

\title{
PERFIL DOS ACIDENTES OFÍDICOS OCORRIDOS NO MUNICÍPIO DE ALTA FLORESTA-MATO GROSSO
}

\author{
Linda Mara Alvino dos Santos Ximenes ${ }^{1}$, Lucas Santos Santana ${ }^{2}$, Oclizio Medeiros \\ das Chagas Silva ${ }^{3}$, Mozarte Santos Santana ${ }^{4}$
}

1. Graduada em Biologia pela UNEMAT, Alta Floresta, Mato Grosso

Brasil.(lindabiologia@hotmail.com)

2. Mestrando em Engenharia Agrícola na UFLA

3. Mestrando em Engenharia Florestal na UFLA

4. Doutorando em Agroquímica na UFLA

Recebido em: 06/04/2018 - Aprovado em: 10/06/2018 - Publicado em: 20/06/2018

DOI: 10.18677/EnciBio 2018A125

\begin{abstract}
A Organização Mundial de Saúde calcula que ocorram no mundo 421.000 a 2,5 milhões de acidentes causados por serpentes venenosas, com 0,02 a 0,1 milhões de mortes anuais. No Brasil o ofidismo remete a um problema de saúde pública pela periodicidade, morbidade e mortalidade que causa. O objetivo deste trabalho foi traçar o perfil clínico epidemiológico dos acidentes ofídicos no município de Alta Floresta-MT. Foram analisados: percentual e gênero das serpentes, total de casos anuais e mensais, área de ocorrência, sexo, idade, ocupação das vítimas, região do corpo mais atingida, circunstâncias, tempo entre 0 acidente e atendimento, classificação, complicações e evolução dos casos. Dos 118 acidentes notificados apenas $(24,6 \%)$ foram identificadas, destes $(22,9 \%)$ causados por serpentes peçonhentas e $(1,7 \%)$ não peçonhentas, a maioria pertencia ao gênero Bothrops $(16,1 \%)$. O ano de maior ocorrência foi 2013 (18,6\%), quanto aos meses predominaram de novembro a abril $(71,2 \%)$. Esses acidentes ocorreram mais na área rural $(66,9 \%)$, no sexo masculino $(73,8 \%)$, entre faixa etária de 20 a 49 anos $(47,5 \%)$, em trabalhadores rurais $(13,7 \%)$, sendo os braços mais afetados $(48,4 \%)$ seguido das pernas (41,5\%). Houve vítima menor de 1 ano $(0,8 \%)$ e óbito em $(2,5 \%)$. Em relação a acidente de trabalho (45,8\%). Foram atendidos em tempo menor que três horas $(62,7 \%)$ dos casos, considerado grau leve $(48,3 \%)$, os quais evoluíram para a cura (83,9\%). Observou se que o perfil epidemiológico desses acidentes se concentra em trabalhadores rurais, entre 20 e 49 anos se acidentando em membros superiores com serpentes do gênero Bothrops.
\end{abstract}

RESUMO

PALAVRAS-CHAVE: Vigilância Epidemiológica, notificação, ocorrência.

\section{PROFILE ACCIDENTS SNAKEBITE OCCURRED IN THE MUNICIPALITY ALTA FLORESTA-MATO GROSSO}

\begin{abstract}
The World Health Organization estimates that 421,000 to 2.5 million accidents occur worldwide in the world caused by poisonous snakes, causing 0,02 to 0,01 million deaths annually. In Brazil, the snake refers to a public health problem due to the
\end{abstract}


periodicity, morbidity and mortality it causes. The purpose this work it was draw the epidemiological clinical profile of accidents caused by snakes in the city of Alta Floresta-MT. Were analyzed: percentage and identification gender of snakes, Total annual and monthly cases, area of occurrence, sex, age, occupation of victims, most affected anatomical region, circumstances, time between the accident and treatment, classification, complications and cases evolution. Of the 118 reported accidents (24.6\%) were identified, of these $22.9 \%$ was caused by venomous snakes and $1.7 \%$ non-venomous snakes, in which the majority belonged to Bothrops genus (16.1\%). The highest year occurrence was in 2013 (18.6\%), while seasonality predominated from November to April (71.2\%). These accidents occurred in the rural area $(66.9 \%)$, in the male $(73.8 \%)$, in the age group $20-49(47.5 \%)$, in rural workers $(13.7 \%)$. the upper limbs were most affected (48.4\%) followed by lower limbs (41.5\%). There was a victim under 1 year $(0.8 \%)$ and death in $(2.5 \%)$. In relation to work accident there was $45.8 \%$. The time elapsed between the bite and care was less than three hours in $62.7 \%$ of the cases, the majority considered mild $(48.3 \%)$, which evolved to cure $(83.9 \%)$. It was observed that the epidemiological profile of the accidents is concentrated in rural workers, between 20 and 49 years of age, occurring in upper limbs with snakes of the genus Bothrops.

KEYWORDS: Epidemiological Surveillance, notification, occurrence.

\section{INTRODUÇÃO}

A ocorrência de acidentes com ofídios constitui problema de Saúde Pública, no qual esses casos geralmente ocorrem em municípios do interior do País. Informações regionais cadastradas e atualizadas são de suma importância para ampliações e aplicações de ações que previnam e evitem a ocorrência desses acidentes (SANTANA et al., 2015).

De acordo com Organização Mundial de Saúde ocorre no mundo cerca de 421.000 a 2,5 milhões de acidentes causados por serpentes peçonhentas, com 0,02 a 0,1 milhões de mortes por ano (GRACIANO et al., 2013). No Brasil, 9 gêneros de serpentes peçonhentas são reconhecidos: Bothrops, Bothropoides, Bothriopsis, Bothrocophia, Rhinocerophis, Crotalus, Lachesis, Micrurus e Leptomicrurus (BARBOSA, 2015). Dentre os acidentes ditos graves, enfatizam-se os causados por serpentes dos gêneros Bothrops, Crotalus, Lachesis e Micrurus, boa parte destes acidentes é fatal na ausência de terapêutica imediata e pertinente (CORREIA et al., 2016).

Diante disso, em 1901 a Vital Brazil iniciou a produção de soro antiofídico e a introdução de um "Boletim para observação dos acidentes". Este boletim era enviado juntamente com as ampolas de soro para ser preenchido e devolvido ao laboratório produtor. Em 1911 publicou o livro "A Defesa contra o Ophidismo" e provou que o tratamento tinha que ser feito com um soro específico, ou seja, para cada tipo de cobra, um tipo de soro antivenenoso (MELGAREJO et al., 2011). As fichas de notificações de animais peçonhentos foram remodeladas no decorrer dos anos, contudo, o Boletim criado por Vital Brazil é a base e o instrumento de coleta que deu origem aos atuais sistemas nacionais de informação para acidentes por animais peçonhentos.

Pesquisas de (BOCHNER; STRUCHINER, 2003) verificaram que o perfil epidemiológico desses acidentes se manteve inalterado ao longo dos anos, sendo mais comuns em pessoas do sexo masculino, trabalhadores rurais, entre 15 a 49 anos, atingindo principalmente as pernas e pés, e a maioria dos acidentes é atribuída às serpentes do gênero Bothrops. 
Até 1985, o soro antiofídico para uso humano não fazia parte do Programa Nacional de Imunizações do Ministério da Saúde (CARDOSO; WHEN, 2003). Uma nova etapa ao controle de acidentes ofídicos surgiu em 1986, quando foi implantado - Programa Nacional de Ofidismo na antiga Secretaria Nacional de Ações Básicas em Saúde do Ministério da Saúde. Os acidentes ofídicos passaram a ser de notificação compulsória no país e se estabeleceu entre as Secretarias Estaduais de Saúde e o Ministério da Saúde um sistema de trocas de soros por informações epidemiológicas (CARDOSO; WHEN, 2003).

Os sistemas nacionais de informação que registram acidentes por ofídios são: SINAN (Sistema de Informação de Agravos de Notificação), SINITOX (Sistema Nacional de Informações Tóxico-Farmacológicas), SIH-SUS (Sistema de Informações Hospitalares do Sistema Único de Saúde), SIM (Sistema de Informações sobre Mortalidade). A coleta desses dados é necessária para criação de campanhas preventivas e orientativas sobre os casos ocorridos regionalmente. Neste contexto, este estudo teve como objetivo traçar o perfil clínico epidemiológico dos acidentes ofídicos ocasionados por serpentes no município de Alta Floresta-MT.

\section{MATERIAIS E MÉTODOS}

A Região de pesquisa compreendeu o município de Alta Floresta-MT Figura 1, no extremo norte do Estado do Mato Grosso. Sua área urbana está situada nas coordenadas geográficas Lat. 952'21.73"S e Long. 56² 4'54.65"O de Greenwich, a $800 \mathrm{~km}$ da capital do Estado (FOSTIER et al., 2015). A extensão territorial é de aproximadamente $8.976,204 \mathrm{Km}^{2}$ e limita-se com os municípios de Tabaporã, Novo Mundo, Juara, Nova Canaã do Norte, Nova Monte Verde, Carlinda, Paranaíta e o estado do Pará. Com uma população estimada no ano de 2017 em 50.189 habitantes (IBGE, 2018a), está posicionada na mesorregião 127, microrregião 519 e coberta por densa floresta tropical (bioma amazônico), a temperatura varia entre 20 을 a $38^{\mathrm{a}} \mathrm{C}$, tendo em média $26^{\circ} \mathrm{C}$. Com clima quente e úmido com quatro meses seco, suas principais características são as elevadas temperaturas podendo chegar a $45^{\circ}$ $\mathrm{C}$ nos dias mais quentes. Köppen adaptado por (ALVARES et al., 2013).

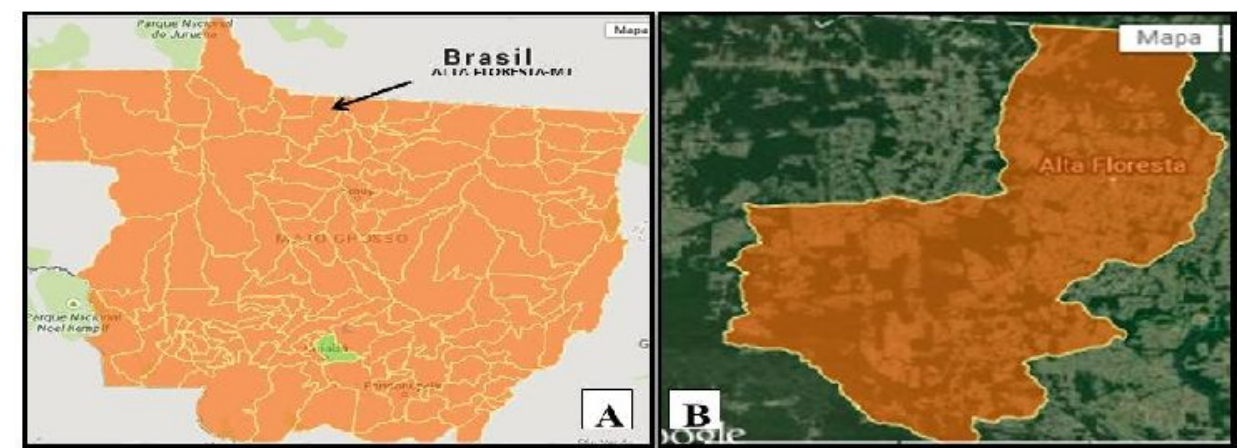

FIGURA 1. Mapa mostrando o Estado de Mato Grosso (A) e o município de Alta Floresta (B).

Para coleta de dados foi realizado um levantamento das características clínico-epidemiológicas dos casos de acidentes ofídicos notificados na Vigilância Epidemiológica (VIGEP) da Secretaria Municipal de Saúde de Alta Floresta-MT, no período entre janeiro de 2007 a dezembro de 2015 com 108 meses analisados.

A VIGEP realiza um trabalho junto ao Hospital Regional de Alta Floresta (HRAF), onde são atendidos os casos de acidentes por animais peçonhentos, incluindo acidentes ofídicos que ocorrem no município. É fornecida à unidade 
hospitalar uma ficha de notificação compulsória para cada ampola específica de soro utilizada. Em casos de acidentes por serpentes não peçonhentas, quando não há necessidade da soroterapia, a ficha de notificação é preenchida da mesma forma, informando que a serpente não é peçonhenta. É obrigatório o preenchimento correto de todos os campos pertencentes na ficha de notificação, caso a vítima esteja impossibilitada de comunicar-se as perguntas são direcionadas ao acompanhante.

Os dados coletados no HRAF são enviados à VIGEP, que repassa ao SINAN e recebe em troca do Ministério da Saúde ampolas de soro, conforme a demanda do município. As características clínico-epidemiológicas analisadas neste trabalho provieram de informações contidas nas fichas individuais dos casos padronizadas pelo SINAN que se encontram arquivadas na VIGEP. A análise da pesquisa foi fundamentada na utilização de dados escolhidos pela ficha de animais peçonhentos (serpentes), calculadas as porcentagens e armazenados em tabelas e planilha do programa Microsoft Excel®, versão 2010.

Foram observadas a quantidade de acidentes ofídicos no município, considerando: percentual de identificação das serpentes, gêneros das serpentes identificadas pela vítima, socorrista e/ou equipe de saúde no atendimento imediato, total de casos anuais e mensais entre 2007/2015, área de ocorrência do acidente: zona rural, área urbana e periférica, sexo e idade das vítimas (masculino ou feminino), ocupação das vítimas (trabalhador, estudante, aposentado), região do corpo mais atingida (pernas e pés, braços e mãos, cabeça e tronco), circunstâncias em que ocorreram os acidentes (trabalho ou lazer), tempo entre o acidente e o atendimento médico, classificação dos casos em leve, moderado ou grave e complicações causadas até a evolução do tratamento.

\section{RESULTADOS E DISCUSSÃO}

De acordo com os resultados, foram notificados e repassados à VIGEP de Alta Floresta-MT no período de estudo, cerca de 118 casos de acidentes ofídicos nos quais houve identificação relacionada ao gênero da serpente em $24,6 \%$ (29 casos) e $75,4 \%$ (89 casos) não foram identificados (Figura 2). A equipe que presta o primeiro atendimento às vítimas, e responsável pelo preenchimento da ficha de notificação dos casos de acidentes é composta por médicos, enfermeiros e técnicos, o que dificulta a identificação das serpentes.

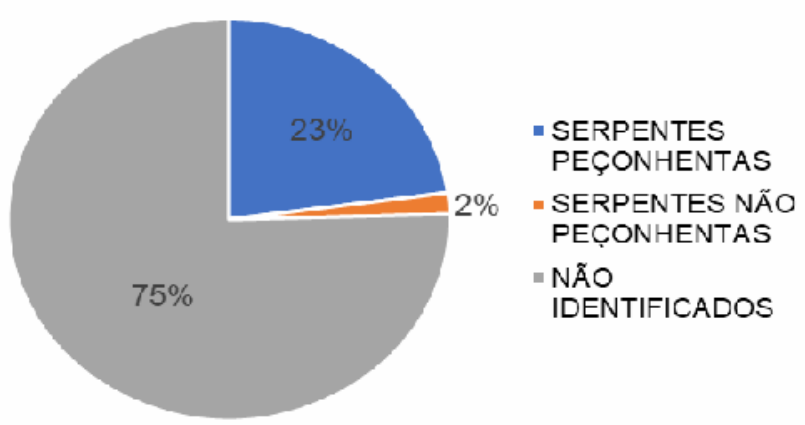

FIGURA 2. Percentual de identificação de serpentes que ocasionaram acidentes ofídicos notificados entre os anos de 2007/2015 em Alta Floresta-MT. 
Além disso, na maioria das ocasiões, o acidentado não leva a serpente na unidade de atendimento. Assim o tratamento é realizado através do diagnóstico, levando em consideração as manifestações clínicas que a vítima apresenta ao ser examinada pelos profissionais. A padronização atualizada de condutas de diagnóstico e tratamento dos acidentados é imprescindível, pois, as equipes de saúde não recebem informações taxonômicas durante os cursos de graduação ou no decorrer da atividade profissional (SILVA et al., 2017). Os dados obtidos concordam com o trabalho de Albuquerque et al., (2013) no qual observaram que as informações de identificação dos gêneros das serpentes foram totalmente ignoradas.

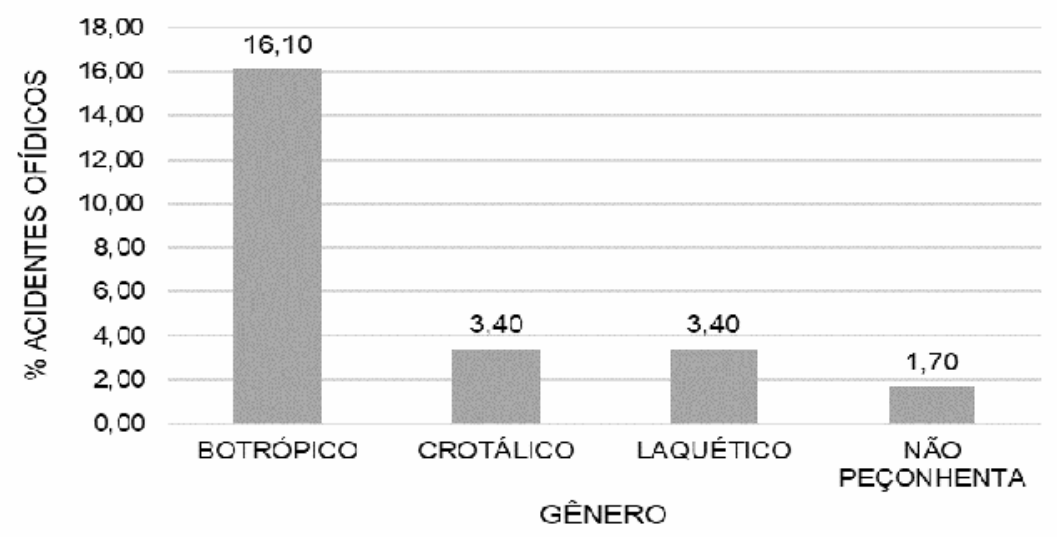

FIGURA 3. Frequência dos acidentes ofídicos no municipio de Alta Floresta-MT no período de 2007/2015, quanto ao gênero da serpente.

$\mathrm{Na}$ (Figura 3), pode-se observar que dos 29 casos que houve identificação dos gêneros de serpentes peçonhentas no município, $16,1 \%$ foram do gênero Bothrops,3,4\% do gênero Crotalus, 3,4\% do gênero Lachesis. As serpentes derivam de caçadores furtivos não construtores, de característica noturna e forrageira, que consumiam presas vertebradas de corpo mole ou invertebrados em ambientes quentes, bem regados e com grande vegetação (DA COSTA et al., 2018). A epidemiologia dos acidentes ofídicos no município segue um padrão que se mantém inalterado nos últimos 100 anos no Brasil, onde a maioria dos casos é atribuída a serpentes do gênero Bothrops (SANTANA et al., 2015;). Bernarde et al. (2015), confirmam que isso pode estar relacionado à boa capacidade de adaptação das jararacas, que são amplamente encontradas em vários ambientes até em áreas urbanas. Wen et al., (2015) concluem em suas pesquisas que Bothrops atrox é a espécie mais frequente envolvida com acidentes ofídicos na região Amazônica. Este Gênero que atualmente é responsável por $90 \%$ dos 20.000 casos anuais reportados (CARDOSO; WHEN, 2003). O alto índice de ataques pode ser estar relacionado com o comportamento desses animais, que durante o dia ficam de forma enrolada ou encurvada no meio de folhas e raramente estão expostas a luz solar, com atividades noturnas e diurnas esses animais são designados de tigmotermos (SAZIMA 1988). 


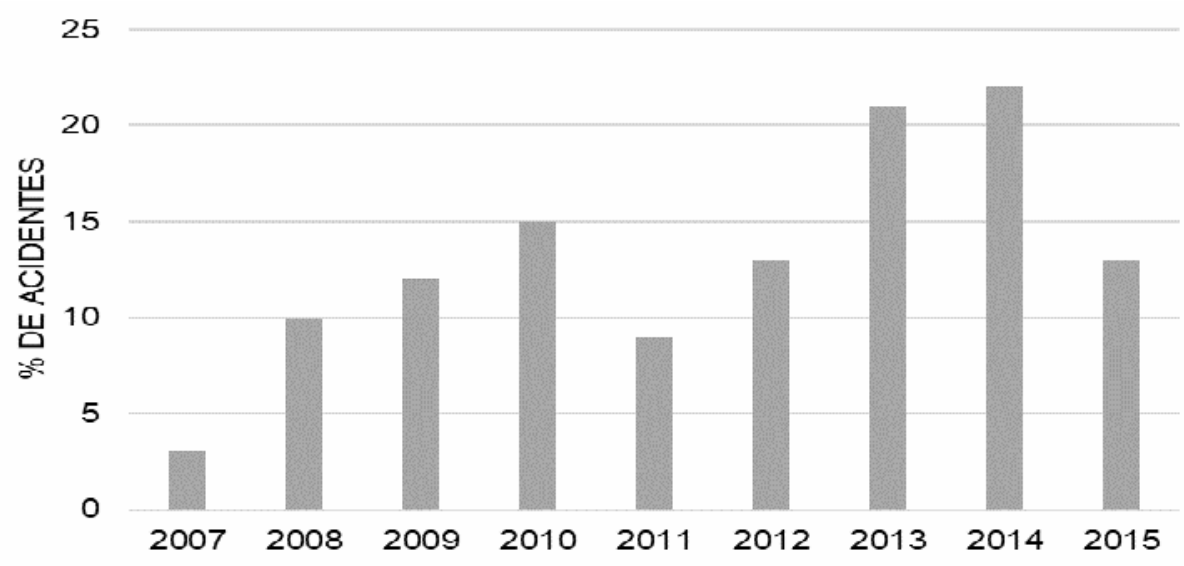

FIGURA 4. Percentual de acidentes por ofídios notificados entre 2007/2015 em Alta Floresta-MT.

A (Figura 4) expõe a distribuição total de acidentes ofídicos ocorridos no município entre os anos de 2007/2015. Em 2007 foram notificados apenas 03 acidentes por ofídios, o que pode estar relacionado à falta de compromisso da unidade de saúde em notificar os casos e enviar dados à VIGEP. O maior número de registros no período da pesquisa foi registrado no ano de 2013 e 2014 com 22 e 21 casos respectivamente. Esses anos coincidem com o monitoramento de resgate de fauna, no processo de construção de UHE no município vizinho, no entanto com pouca estrutura para realizar atendimentos médicos os casos são levados para alta floresta. Segundo relatório básico ambiental da UHE Teles Pires, as atividades foram realizadas em três etapas: Durante o acompanhamento das ações de supressão de vegetação nas áreas de implantação das estruturas permanentes e instalações de apoio; durante o acompanhamento das ações de supressão de vegetação das áreas de formação do reservatório e durante o acompanhamento do enchimento do reservatório que são justamente entre os anos de 2013 e 2014.

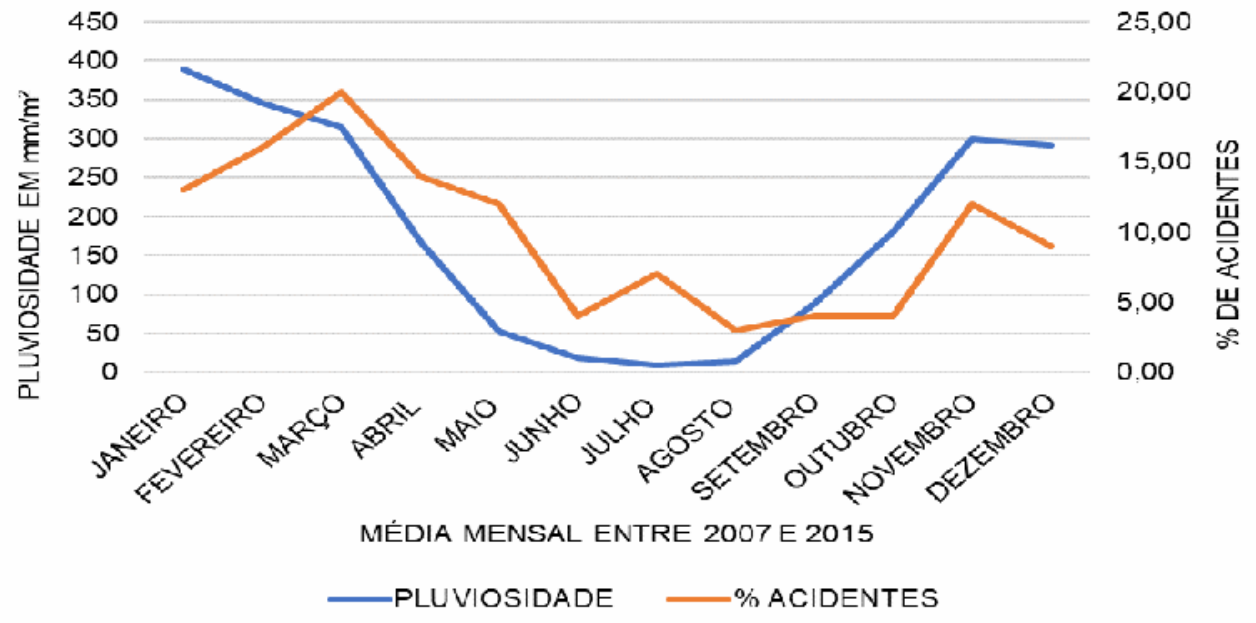

FIGURA 5. Percentual de acidentes por ofídios notificados entre 2007/2015 em Alta Floresta-MT, relacionando com a quantidade de chuvas da região expressada em $\mathrm{mm} / \mathrm{m}^{2}$ (fonte séries view INPE)

A (Figura 5), destaca que a distribuição de acidentes ofídicos ocorridos no período referente aos meses entre janeiro de 2007 a dezembro de 2015, estão comumente relacionadas aos períodos chuvosos. Mesmo de forma irregular houveram acidentes por ofídios durante todos os meses do ano, no qual, a maioria dos acidentes ocorreram nos meses de novembro a abril, como o observado nesta ENCICLOPÉDIA BIOSFERA, Centro Científico Conhecer - Goiânia, v.15 n.27; p.1463 
pesquisa e também em trabalhos de autores como (BERNARDE et al., 2015; SANTOS et al., 2012; LOPES et al., 2017). O elevado número de acidentes registrados pode estar ligado com a época de maior manejo agropecuário.

O alto índice pluviométrico desse período faz com que o produtor se envolva mais com trabalho de campo, principalmente no controle de plantas daninhas em regiões de forte expressão na pecuária. Segundo Lemos et al. (2009) o grande índice de ataques está relacionado a época do ano com maiores índices pluviométricos, onde se intensificam a atividade agrícola, propiciando assim, ao encontro com a serpente.

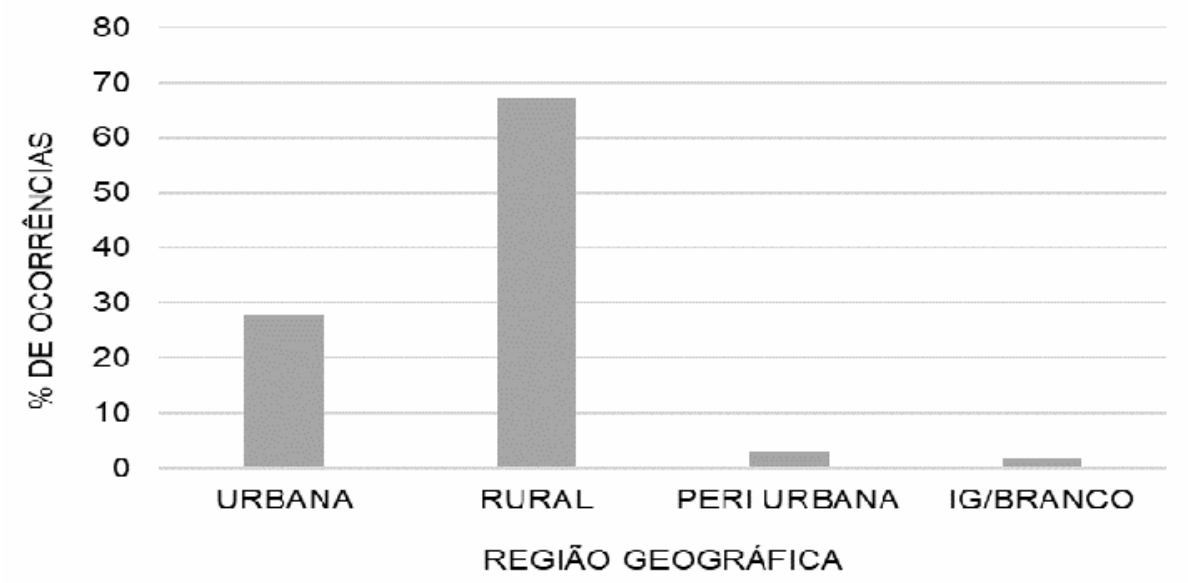

FIGURA 6. Área de ocorrência dos acidentes entre 2007/2015 no município de Alta Floresta-MT, divididos em zona rural, urbana e periferia-urbana.

$\mathrm{Na}$ (Figura 6), nota-se que $66,9 \%$ dos casos ocorreram na zona rural, $28 \%$ na zona urbana e $3,4 \%$ na zona periférica-urbana e 1,7\% dos dados foram ignorados e brancos. A ocorrência do acidente ofídico está, em geral, relacionada a fatores climáticos, como pluviosidade e temperatura e também com o envolvimento do trabalho no campo, onde geralmente se trabalha próximo ao habitat natural da serpente, causando contato direto com o animal Silva et al. (2017), em decorrência disto, a maioria dos acidentes acontecem na zona rural, como o observado nesta pesquisa, e também por autores como (ALBUQUERQUE et al., 2013; CARMO et al., 2016; JESUS et al., 2016; SILVA et al., 2017).

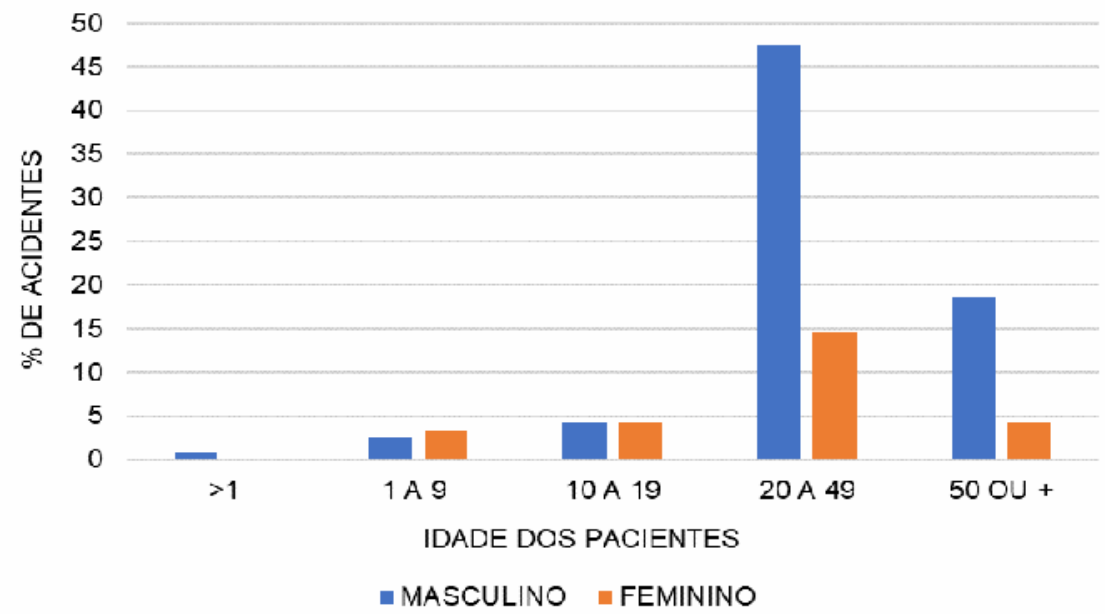

FIGURA 7. Distribuição dos acidentes ofídicos registrados no município de Alta Floresta-MT entre 2007/2015 quanto à faixa etária das vítimas do sexo masculino e feminino. 
Conforme apresentado na (Figura 7), a maioria dos acidentes ocorreram em pessoas do sexo masculino (73,7\%), e entre as mulheres foram (26,3\%). Nos casos ocorridos entre as mulheres, o maior número de acidentes foi na faixa etária entre 20 a 49 anos com 17 casos. Nos homens a maioria dos acidentes também ocorreu na faixa etária entre 20 a 49 anos, com 56 casos, esses dados estão relacionados ao forte envolvimento do sexo masculino no serviço braçal, em que provocam acidentes ao trabalhar em serviços de fazendas. Outro índice importante é a quantidade de acidentes na faixa etária entre 50 a 79 anos, somando 22 casos (18,6\%).

Alguns autores expõem resultados indicando uma maior relação do sexo masculino e acidentes com serpentes (GRACIANO et al., 2013; SOUSA et al., 2013; DA SILVA et al., 2015). Essa relação já registrada por vários autores se deve ao fato provavelmente pela grande inserção masculina nos trabalhos manuais (ALBUQUERQUE et al., 2013).

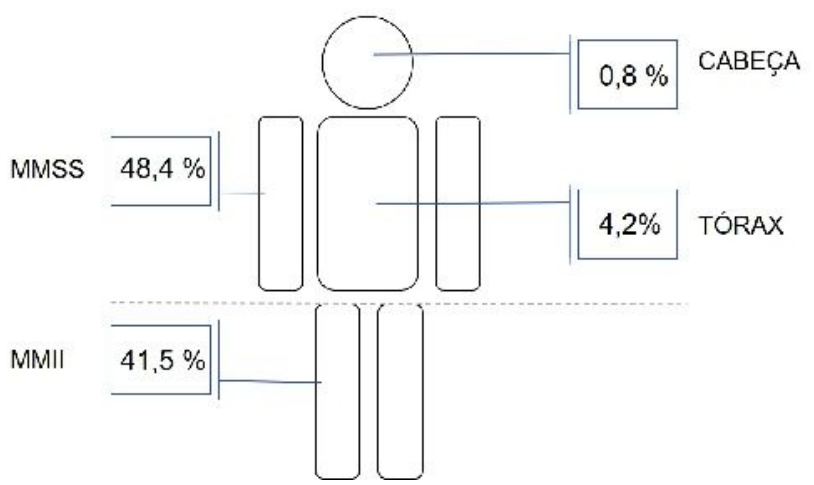

FIGURA 8. Distribuição dos acidentes ofídicos registrados no Município de Alta Floresta-MT, entre 2007/2015 quanto à região anatômica atingida.

Como visto na (Figura 8), as regiões anatômicas mais atingidas por ofídios, tanto em homens quanto mulheres, $48,4 \%$ foram nos membros superiores (MMSS). Nos membros inferiores (MMII) ocorreram 41,5\%, tronco 4,2\% cabeça 0,8\%, e ignorado/branco $5,1 \%$. Foi observado nesta pesquisa, um maior número de acidentes nos MMSS, resultados que não corroboram com a pesquisa feita por outros pesquisadores (SANTOS et al., 2012; ALBUQUERQUE et al., 2013; LEITE, 2013), onde a maior incidência das picadas fora nos MMII. Uma das explicações para o alto índice de ataques no MMSS, pode ser a presença de indústrias madeireiras na região, onde trabalhadores, fazem o manejo manual das peças de madeira. Isso pode ter uma relação estreita com os trabalhos realizados nas coletas a campo da UHE Teles Pires, nos anos de 2013 e 2014. A alta porcentagem de ataques nos MMII está diretamente relacionada com trabalhadores rurais, que exploram trabalhos como: roçagem manual, aplicação de herbicidas e manejo de gado, deixando clara a alta dependência econômica da região no setor agropecuário.

TABELA 1. Demonstração das vítimas que sofreram acidentes ofídicos, quanto a sua ocupação.

\begin{tabular}{lcc}
\hline Ocupação & Números 2007/2014 & \% Casos \\
\hline Pescador profissional & 2 & 1,7 \\
Operador de máquinas & 3 & 2,5 \\
Serviços gerais & 14 & 11,9 \\
Estudantes & 12 & 10,2 \\
Dona de casa & 11 & 9,4
\end{tabular}


Construtor e eletricista

Motorista

Agricultor

Ignorado/branco

Das vítimas acidentadas quanto a ocupação profissional foram notificados conforme (Tabela 1). Onde a maior incidência de acidentes foi observado em trabalhadores rurais. Que também foram encontrados por outros autores, que afirmam: tais atividades levam a alterações do meio ambiente, resultantes do desmatamento para cultivo e formação de pastagem para o gado. Essas mudanças, por si só, já são importantes definidoras do aumento dos acidentes com animais peçonhentos, uma vez que há a invasão do habitat natural dos mesmos (ALBUQUERQUE et al., 2013). O segundo catalogado como alto índice de ataques foram os estudantes, que estão envolvidos em pesquisas e levantamento de fauna (SILVA et al., 2017).

Alguns os casos de acidentes ofídicos também foram identificados quanto a ausência ou uso incorreto de EPI contabilizando 55 casos. Nas pesquisas de Silva et al., (2017) foram observados que acidentes ofídicos acometem principalmente trabalhadores que não usam adequadamente equipamentos de proteção individual (EPI). Mesmo com a proteção de EPIs, Abrahão et al. (2017) atenta o alto risco exposto por trabalhadores no campo. Em seu trabalho realizado em cultura de abacaxizeiro, testou diferentes equipamentos de proteção e concluiu que muitos são ineficientes ao ataque de serpentes. Seus resultados destacam a necessidade de equipamentos específicos ao uso agrícola.

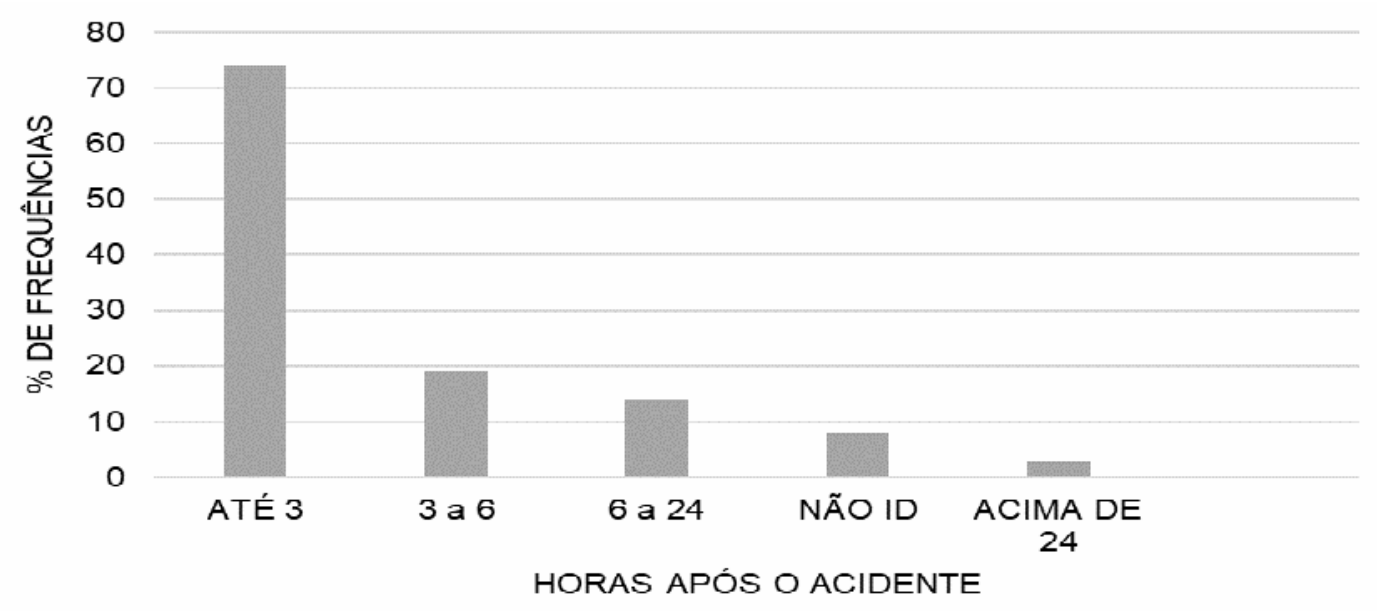

FIGURA 9. Frequência dos acidentes ofídicos no municipio de Alta Floresta-MT, segundo o tempo decorridodo momento do acidente até o atendimento médico,no período de 2007/2015.

A (Figura 9) demonstra a relação do tempo entre a ocorrência e o atendimento das vítimas, onde $62,7 \%$ foram em tempo inferior a 03 horas e $16,1 \%$ dos casos foram atendidos entre 03 e 06 horas após o acidente. Pessoas atendidas entre 06 e 24 horas foram 11,9\% e somente 2,5\% dos casos foram atendidos após 24 horas do acidente, e notificações ignorado/branco foram 6,8\%. O tempo decorrido entre $O$ acidente e 0 atendimento inferior as três primeiras horas observado nesta 
pesquisa, também é corroborada em outras regiões (Lemos et al., 2009). É essencial que o atendimento ocorra no mínimo de tempo possível, a fim de evitar complicações ou mesmo óbito (MACHADO et al., 2016).

Atendimentos que ocorreram em tempo superior a 12 horas após o acidente foram observados por Mise et al. (2007) Isto ocorre devido às condições geográficas próprias da região amazônica (rios, estradas vicinais em péssimas condições, dependência de transporte fluvial ou terrestre) e também pode estar relacionada à resistência do próprio acidentado em buscar atendimento médico necessário (LEITE et al., 2013).

Foi também constatado por Albuquerque et al. (2013) no período de janeiro de 1995 a dezembro de 2003 no Núcleo Regional de Saúde da Paraíba dentre os acidentados, que 86,6\% não apresentaram informações quanto a este dado.

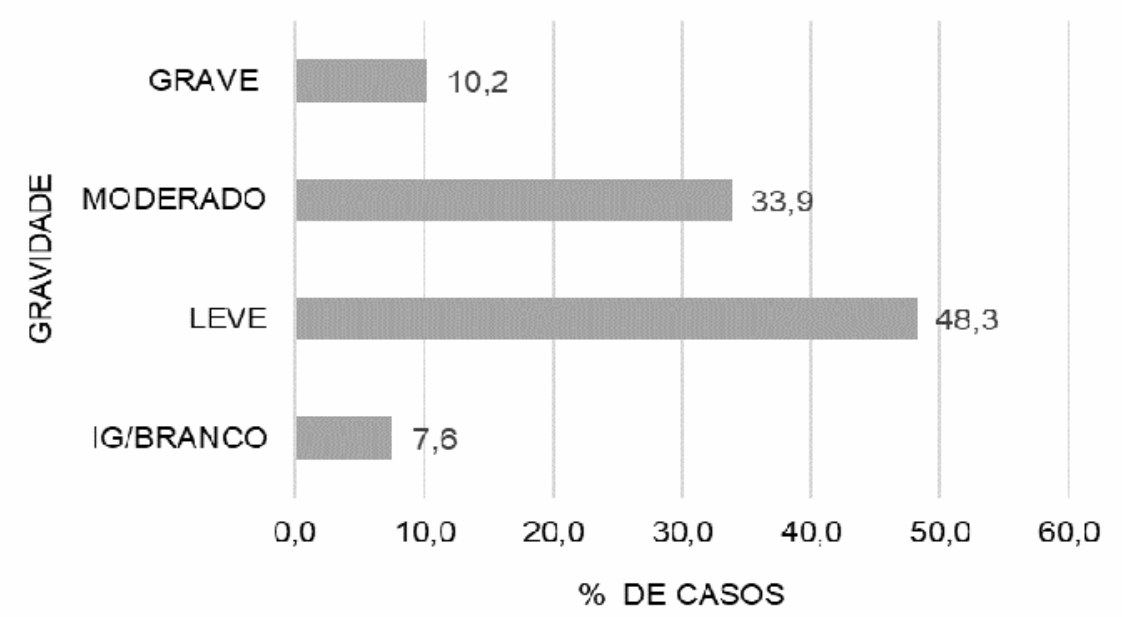

FIGURA 10. Frequência anual dos acidentes ofídicos no município de Alta FlorestaMT, segundo a classificação dos casos (CC) no período de 2007 a 2014/1.

A (Figura 10) representa os resultados segundo a classificação dos casos em grau leve com 48,3\%, moderado $33,9 \%$, grave $10,2 \%$ e ignorado/branco $7,6 \%$. A maior incidência de classificação dos casos para grau leve observado nesta pesquisa também é corrobora com pesquisas de Sousa et al. (2013), onde analisou aspectos epidemiológicos dos acidentes ofídicos no município de Mossoró-RN, no período de 2004 a 2010 e descreveu que 58,7 \% dos acidentes foram identificados como leve, em que os sintomas observados foram dores de cabeça e edema superficial.

Dentre as vítimas de acidentes ofídicos atendidas, foi realizado exame laboratorial para verificar o tempo de coagulação sanguínea (TC) entre janeiro de 2007 a junho de 2014 em apenas $21,4 \%$ dos casos, o tempo de coagulação (TC) deve ser realizado sempre que houver suspeita de acidente botrópico. Segundo Antonussi et al. (2016), a manifestação clínica do acidente botrópico caracteriza-se pela marca das presas no local da picada, sangramento local e edema precoce, possuindo o veneno ação coagulante, proteolítica e vasculotóxica. Segundo Cardoso et al., (2003), o TC prolongado, mesmo sem alterações no local da picada, indica envenenamento e necessidade de administração do soro antiofídico. A determinação do Tempo de Coagulação (TC) é importante medida auxiliar no diagnóstico do envenenamento e acompanhamento dos casos. No entanto, este exame não foi realizado na admissão da maioria das vítimas por ofídios, como o observado nesta pesquisa e também na pesquisa de Lopes et al. (2017). O TC é um ENCICLOPÉDIA BIOSFERA, Centro Científico Conhecer - Goiânia, v.15 n.27; p.1467 2018 
teste simples e rápido, bastante útil e que pode ser utilizado na ausência de equipamentos para realização do coagulograma (BRASIL, 2009a).

Conforme a Tabela $315,3 \%$ dos casos obtiveram resultado de tempo de coagulação normal, e 6,1\% apresentaram resultados alterados. No período entre Julho de 2014 a Dezembro de 2015 estes dados não foram informados à Vigilância Epidemiológica.

TABELA 3. Frequência anual dos acidentes ofídicos no município de Alta FlorestaMT, classificados em: coagulação normal, e uso de soroterapia, no período de 2007 a $2014 / 1$.

\begin{tabular}{cccc}
\hline & Coagulação normal \% & Soroterapia \% \\
\hline Ig/branco & 23,5 & Ig/branco & 2,5 \\
Normal & 15,3 & Sim & 86,4 \\
Alterada & 6,1 & Não & 13,6 \\
\hline
\end{tabular}

Quanto ao uso de soroterapia foi adiministrado soro em $83,1 \%$ das vítimas atendidas. Apenas 14,4\% não receberam o soro, esse prodedimento deve orientado com cautela, pois aplicação errada pode causar danos maiores. Sousa et al. (2011) e Lopes et al. (2017) salientam que como a soroterapia é específica, o reconhecimento das manifestações clínicas é um importante referencial para o diagnóstico do tipo de envenenamento, e uma falha neste, pode comprometer o sucesso do tratamento ao paciente.

O sintomas de ataques de serpentes, pouco diferenciam, portanto vários aspectos devem ser considerados para tal análise. A Tabela 4 dá exemplos dos sintomas gerais apresentados pelos pacientes, observa-se que dos sinais e sintomas observados, 7 casos apresentaram complicações locais (5,9\%), e 98 casos não apresentaram complicações (83,1\%). Segundo Bernarde et al. (2015) A diferenciação do envenenamento laquético do botrópico é relativamente mais difícil devido à semelhança entre os sintomas, caso a serpente causadora não tenha sido capturada e levada até o hospital. O reconhecimento das manifestações clínicas e a história epidemiológica do acidente permitem 0 diagnóstico do tipo de envenenamento (BRASIL, 2009b). Vitímas que apresentaram infecção secundária foram apenas 11 casos $(11,9 \%)$.

TABELA 4. Demonstração de complicações causadas por acidentes ofídicos da picada até a evolução dos casos, no municipio de Alta Floresta-MT entre $2007 / 2015$

\begin{tabular}{lccccc}
\cline { 2 - 6 } & Sim \% & Não \% & Ig/Branco \% & Cura \% & Óbito \% \\
\hline Complicações & 5,9 & 83,1 & 11 & - & - \\
Infecção & 11,9 & 7,6 & 80,5 & - & - \\
Necrose & 0,8 & 6 & 93,2 & - & - \\
Amputação & - & 16,9 & 83,1 & - & - \\
Evolução & - & - & 13,6 & 83,9 & 2,5 \\
\hline
\end{tabular}

A falta do preenchimento correto da ficha de notificação impossibilitou a precisão e e acurácia de alguns dados. De acordo com os dados 6,8\% indicaram que não houve necrose tecidual e/ou amputação de membros em nenhuma das vítimas, e $83,1 \%$ dos dados foram ignoradas/branco em ambos os casos. 
Segundo os resultados da pesquisa 99 vítimas evoluiram para cura $(83,9 \%)$,dados ignorado/branco foram 16 casos $(13,6 \%)$ e vítimas que foram a óbito 3 casos $(2,5 \%)$, em relação a letalidade dos acidentes, dados semelhantes foram encontrados por Silva et al. (2017).

Esse fato demonstra que se o paciente for atendido em curto prazo de tempo e for feito um diagnóstico correto, com profissional capacitado, a chance é alta de o paciente não ter sequelas dos ataques desses animais peçonhentos. Isso claro irá depender da espécie em questão e dos procedimentos adotados.

\section{CONCLUSÃO}

Conforme os resultados apresentados observou-se que o perfil clínico epidemiológico de ataques no município de Alta Floresta-MT é composto por ataques de serpentes do gênero Bothrops, se concentrando geograficamente na zona rural, onde a maioria dos acidentados foram pessoas do sexo masculino, trabalhadores rurais na faixa etária entre 20 a 49 anos e ataques concentrados nos principalmente em membros superiores, porém com altos ataques em membros inferiores.

A inconsistência de dados relacionada ao tipo de serpente causadora do acidente foi de $75,4 \%$, demonstrando a importância da inclusão de profissionais capacitados na área da saúde para identificação de espécies responsáveis pelos acidentes ofídicos.

Esses resultados alertam a secretária de saúde do município ao treinamento de profissionais para melhor preenchimento de fichas e realização de campanha preventivas em regiões de zona rural, orientando e explicando sobre os cuidados a serem tomados em relação principalmente, aos acidentes causados por serpentes do gênero Bothrops.

\section{REFERÊNCIAS}

ABRAHÃO, R. F.; GONZAGA, M. C.; QUEIRÓZ, F. R. C.; TERESO, J. A. Personal Protective Equipment Design and Ergonomic Work Analysis: Protecting Farm Pineapple Growers. The Japanese Journal of Ergonomics, v. 53, p. 552-555, $2017 . \quad$ Disponível em:

https://www.jstage.jst.go.jp/article/jje/53/Supplement2/53_S552/_article/-char/en>. Doi: https://doi.org/10.5100/jje.53.S552

ALBUQUERQUE, P. L. M. M.; JUNIOR, G. B. S.; JACINTO. C. N.; LIMA, C. B.; LIMA, J B. et al. Perfil epidemiológico dos acidentes por picada de cobra em região metropolitana do nordeste do Brasil. Revista do Instituto de Medicina Tropical de São Paulo, v. 55, n. 5, p. 347-51, 2013. Disponível em: <https://wwwscopus.ez26. periodicos.capes.gov.br/record/display.uri?eid=2-s2.0-

84884136983\&origin=inward\&txGid=6026df6cafe4a5f67340d4639de773a0> Doi: $10.1590 /$ S0036-46652013000500009

ALVARES, C. A.; STAPE, J. L.; SENTELHAS, P. C.; De MORAES GONÇALVES, J. L.; SPAROVEK, G. Köppen's climate classification map for Brazil. Meteorologische Zeitschrift, v. 22, n. 6, p. 711-728, 2013. Disponível em: http://www.ingentaconnect.com/content/schweiz/mz/2013/00000022/00000006/art00 008>.https://doi.org/10.1127/0941-2948/2013/0507 
ANTONUSSI, T. D.; RODRIGUES, F. R.; SILVA, G. M. A.; SALVADOR, R. C. L.; NARDO, C. D. D. et al. Acidentes causados por serpentes do gênero Bothrops em pequenos animais - revisão de literatura. Nucleus Animalium, v. 8, n. 2, p. 4, 2016. Disponível

em: http://www.nucleus.feituverava.com.br/index.php/animalium/article/view/1597. Doi: http://dx.doi.org/10.3738/1982.2278.1597

BARBOSA, I. R. Aspectos Clínicos e Epidemiológicos dos acidentes provocados por animais peçonhentos no Estado do Rio Grande do Norte. Revista Ciência Plural. 1(3):2-13, $2015 . \quad$ Disponível em:< https://periodicos.ufrn.br/rcp/article/view/8578/6179>

BERNARDE, P. S.; MOTA DA SILVA, A.; ABREU, L. C. Ofidismo no Estado do Acre - Brasil. Journal of Amazon Health Science, v. 1, n. 2, p. 44-63, 2015. Disponível em: < https://revistas.ufac.br/revista/index.php/ahs/index

BOCHNER, R.; STRUCHINER, C. J. Epidemiologia dos acidentes ofídicos nos últimos 100 anos no Brasil: uma revisão. Cadernos de Saúde Pública, v.18, p.735746, 2003. Disponível em: <http://www.scielo.br/pdf/\%0D/csp/v19n1/14900.pdf>

BRASIL. Ministério da Saúde. Secretaria de Atenção à Saúde. Departamento de Atenção Básica. Vigilância em saúde: zoonoses. Caderno de Atenção Básica, pag. 117- Brasília, 2009a. Disponível em:

http://bvsms.saude.gov.br/bvs/publicacoes/vigilancia_saude_zoonoses_p1.pdf>

BRASIL. Ministério da Saúde. Secretaria de Vigilância em Saúde. Departamento de Vigilância Epidemiológica. Guia de vigilância - 7. Ed, p. 787 - Ministério da Saúde. Brasília, 2009b. Disponível em: http://bvsms.saude.gov.br/bvs/publicacoes/guia_vigilancia_epidemiologica_7ed.pdf

CARDOSO, J. L.; WHEN F. H. Animais peçonhentos no Brasil: biologia, clínica e terapêutica dos acidentes. Cap. 1, pag. 4-5. São Paulo, 2003. Disponível em: < https://www.researchgate.net/publication/247853352_Animais_Peconhentos_no_Bra sil_Biologia_clinica_e_terapeutica_dos_acidentes>. DOI: $10.1590 /$ S0036$4 \overline{6} \overline{6} 520030 \overline{0} 06000 \overline{0}$

CARMO, E. A.; NERY, A. A.; DE JESUS, C. S.; CASOTTI, C. A. Internações hospitalares por causas externas envolvendo contato com animais em um hospital geral do interior da Bahia, 2009-2011. Epidemiologia e Serviços de Saúde, v.25, n.1, n.105-114, 2016. Disponível em: <https://doi.org/10.5123/S167949742016000100011>. doi: 10.5123/S1679-49742016000100011

CORREIA, F. F; SILVA, M. P. L; SOUZA, M. R; FONTES, C. J. F. Perfil ClínicoEpidemiológico dos Acidentes Ofídicos Ocorridos no Município de Cacoal, Rondônia, Brasil, 2011 e 2015. Revista Eletrônica FACIMEDIT, v. 5, n. 2, 2016. Disponível em:<http://www.facimed.edu.br/o/revista/pdfs/bd6c8fb1044c1ca57910243f37ce69fb. pdf>

DA COSTA, C, R.; DA ROCHA, D. G.; DA SILVA, W. D. A desmistificação das cobras na educação: dos perigos iminentes aos usos inteligentes. LINK SCIENCE 
PLACE - Interdisciplinary Scientific Journal, v. 4, n. 4, 2018. Disponível em: $<$ http://revista.srvroot.com/linkscienceplace/index.php/linkscienceplace/article/view/4 23/216>

DA SILVA, A. M.; BERNARDE, P. S.; DE ABREU, L. C. Accidents with poisonous animals in Brazil by age and sex. Jornal of Human Growth and Development, v. 25, n.1, p. 54-62, 2015. Disponível em: < http://www.journals.usp.br/jhgd/article/view/96768>. dx.doi.org/10.7322/jhgd.96768

FOSTIER, A. H.; MELENDES-PEREZ, J. J.; RICHTER, L. Litter Mercury deposition in the Amazonian rainforest. Institute of Chemistry, University of Campinas, Box 6154, 13083-970, 2015. Disponível em: https://doi.org/10.1016/j.envpol.2015.08.010.

GRACIANO, S. A.; COELHO, M. J.; TEIXEIRA, A. O.; SILVA, J. C. S.; PEREIRA, S. R. M.; FERNANDES, R. T. P. Perfil epidemiológico dos acidentes ofídicos em homens. Revista de Enfermagem Referência, III Série - n. 10, p. 90-94, 2013. Disponível em:<http://www.redalyc.org/articulo.oa?id=388239969016>

IBGE, Instituto Brasileiro de Geografia e Estatísticas, 2018a. Disponível em < https://cidades.ibge.gov.br/brasil/mt/alta-floresta/panorama> Acesso em 20/03/2018.

IBGE, Instituto Brasileiro de Geografia e Estatísticas, 2018b. Disponível em<https://cidades.ibge.gov.br/comparamun/compara. php?lang=\&coduf=51\&idtema $=159 \& \mathrm{cod} v=\mathrm{v} 01 \&$ search $=$ mato-grosso\%7Cjuara\%7Csintese-das-informacoes $-2015>$ Acesso em 20/03/2018.

JESUS, A. G.; GOMES, H.; BARASUOL, A. M.; VIEIRA, G. D. S.; SOUSA, E. R. M. Avaliação dos acidentes ofídicos na região sob influência da usina hidrelétrica Belo Monte - Estado do Pará. Revista Cereus, v. 8, n. 3, p. 02-18, 2016. Disponível em: < http://ojs.unirg.edu.br/index.php/1/article/view/1380/483> Doi: 10.18605/2175-7275

LEITE, R. S.; TARGINO, I. T. G.; LOPES, Y. A. C. F.; BARROS, R. M.; VIEIRA, A. A. Epidemiologia dos acidentes ofídicos ocorridos nos municípios do Estado da Paraíba, Brasil. Ciência \& Saúde Coletiva, v. 8, n. 5, p. 1463-71, 2013. Disponível em: http://dx.doi.org/10.1590/S1413-81232013001100032>

LEMOS, J. C.; De ALMEIDA, T. H.; LIA FOOK, S. M.; PAIVA, A. A.; SIMÕES, M. O. $S$. Epidemiologia dos acidentes ofídicos notificados pelo Centro de Assistência e Informação Toxicológica de Campina Grande (Ceatox-CG), v. 12. n. 1 p. 09-50, 2009. Disponível em: <https://doi.org/10.1590/S1415790X2009000100006>

LOPES, A. B; OLIVEIRA, A. A.; DIAS, F. C. F.; De SANTANA, V. M. X.; OLIVEIRA, V. S. et al. Perfil epidemiológico dos acidentes por animais peçonhentos na região Norte entre os anos entre 2012 e 2015: uma revisão. Revista de Patologia do Tocantins, $\quad$ v. $4 \quad$ n. $2, \quad 2017 . \quad$ Disponível em: <https://sistemas.uft.edu.br/periodicos/index.php/patologia/article/view/3753>. Doi: 10.20873/uft.2446-6492.2017v4n2p36. 
MACHADO, C.; LEMOS, E. R. S. Ofidismo no Estado do Rio de Janeiro, Brasil, no período de 2007-2013. Revista Eletrônica Estácio Saúde, v. 5, n. 2, p. 67-77, 2016.

http://revistaadmmade.estacio.br/index.php/saudesantacatarina/index>

MELGAREJO, A.; PUORTO, G.; COTTA, G.; CASAIS, L.; LIRA, R.; BOCHNER, R.; MISE, Y. Manual do Curso Serpente e Ofidismo. Pág. 8. Salvador- BA, 2011. Disponível em: <https://redevitalparaobrasil.files.wordpress.com/2011/03/manualcurso_serpentes_ofidismo2011.pdf>.

MISE, Y. F.; SILVA, R. M. L. S.; CARVALHO, F. M. Envenenamento por serpentes do gênero Bothrops no Estado da Bahia: aspectos epidemiológicos e clínicos. Revista da Sociedade Brasileira de Medicina Tropical. 40(5), pag. 571, Salvador, 2007. em:

http://www.ingentaconnect.com/content/doaj/00378682/2007/00000040/00000005/art 00015>. https://doi.org/10.1590/S0037-86822007000500015

SANTANA, V. T. P.; SUCHARA, E. A. Epidemiologia dos acidentes com animais peçonhentos registrados em Nova Xavantina - MT. Revista de Epidemiologia e Controle de Infecção, v. 5, n. 3, p. 141-146, 2015. Disponível em: http://online.unisc.br/seer/index.php/epidemiologia/article/view/5724 doi:http://dx.doi.org/10.17058/reci.v5i3.5724.

SANTOS, G. J.; SILVEIRA C.; MARTINS, M. B. Epidemiologia dos acidentes causados por serpentes peçonhentas no estado do Rio Grande do Sul no período de 2007 a 2010, Brasil. pag. 9, Porto Alegre, 2012. Disponível em: http://hdl.handle.net/10183/72397

SAZIMA, I. Um estudo de biologia comportamental da jararaca, Bothrops jararaca, com uso de marcas naturais, MEN Instituto Butantan, v. 50, n. 3, p. 83-99, 1988.

SILVA, J. Perfil epidemiológico dos acidentes com animais peçonhentos em Tangará da Serra-MT, Brasil (2007-2016). Journal Health Npeps, v. 2, n. 1, p.5-15, 2017. Disponível <https://periodicos.unemat.br/index.php/jhnpeps/article/view/1797/1651>.

SOUSA, R. S.; COSTA, K. M. F. M.; CAMARA, I. M. B.; De OLIVEIRA, G. B.; De MOURA, S. R. et al. Aspectos Epidemiológicos dos Acidentes Ofídicos no Município de Mossoró, Rio Grande do Norte, no período de 2004 a 2010. Revista de Patologia Tropical v. 42, p. 107-110, 2013. Disponível em: <https://www.revistas.ufg.br/iptsp/article/view/23593>.

Doi:https://doi.org/10.5216/rpt.v42i1.23593

SOUSA, C. S. A.; NUNES, R. O. Epidemiologia dos Acidentes Ofídicos no município de Rolim de Moura, Rondônia. Revista Científica Facimedit, v 3, n. 3, p. 252, 2011. Disponivelem<http://www.facimed.edu.br/o/revista/pdfs/9fdbd7663aebbf66d048fd14d 2b6dd23.pdf>.

WEN, F.H.; MONTEIRO, W.M.; SILVA, A. M. M.; TAMBOURGI, D. V.; SILVA, I. M. et al. Snakebites and scorpion stings in the Brazilian amazon: identifying research priorities for a largely neglected problem. Plos Neglected Tropical Diseases, v. 9, 
n. 5,2015 p. $11, \quad$ Disponível em: < http://www.ncbi.nlm.nih.gov/pmc/articles/PMC4440781/pdf/pntd.0003701.pdf > Doi: 10.1371/journal.pntd.0003701. 